\title{
Efficacy and Safety of Lacosamide in Adolescents with Lennox-Gastaut Syndrome
}

\author{
Han Som Choi, MD ${ }^{1,2}$, Se Hee Kim, MD²,3, Hoon-Chul Kang, MD², Joon Soo Lee, MD², \\ Heung Dong Kim, MD ${ }^{2,3}$ \\ 'Department of Pediatrics, Chungnam National University Hospital, Daejeon, Korea \\ ${ }^{2}$ Department of Pediatrics, Yonsei University College of Medicine, Seoul, Korea \\ ${ }^{3}$ Division of Pediatric Neurology, Department of Pediatrics, Severance Children's Hospital, Seoul, Korea
}

Received: May 4, 2020

Revised: May 26, 2020

Accepted: May 26, 2020

Corresponding authors:

Heung Dong Kim, MD

Department of Pediatrics,

Severance Children's Hospital,

Epilepsy Research Institute, Yonsei

University College of Medicine,

50-1 Yonsei-ro, Seodaemun-gu,

Seoul 03722, Korea

Tel: +82-2-2228-2050

Fax: +82-2-393-9118

E-mail: hdkimmd@yuhs.ac

Se Hee Kim, MD

Department of Pediatrics,

Severance Children's Hospital,

Epilepsy Research Institute, Yonsei

University College of Medicine,

50-1 Yonsei-ro, Seodaemun-gu,

Seoul 03722, Korea

Tel: +82-2-2228-2050

Fax: +82-2-393-9118

E-mail: seheekim@yuhs.ac
Purpose: We aimed to assess the efficacy and safety of lacosamide in Korean adolescents with Lennox-Gastaut syndrome (LGS), especially in those who concomitantly used other sodium channel blockers (SCBs).

Methods: We retrospectively reviewed the medical records of adolescents with LGS who initiated lacosamide from ages 16 to 18 . The efficacy of lacosamide was evaluated by seizure frequency before and after lacosamide trial. Safety was assessed by lacosamide-related adverse events, consequent dosage titration, and titration effects. We compared the efficacy and safety of lacosamide according to concomitant use of other SCBs.

Results: In 26 eligible adolescents with LGS, the median age of seizure onset was 2.0 years, and the median age of lacosamide initiation was 17.1 years. At the time of lacosamide initiation, the median number of concomitant antiepileptic drugs was 4 , and 23 patients (88\%) had tried dietary, surgical, or neuromodulatory therapies. Patients were on lacosamide for a median of 13.5 months with a median maximal dosage of $8.1 \mathrm{~kg} / \mathrm{mg} /$ day. After lacosamide trial, 11 patients $(42 \%)$ had an over $50 \%$ reduction of seizures. Six patients (23\%) had lacosamide-related adverse events. The percentage of patients on concomitant SCBs was higher among non-responders (10 of $15,67 \%$ ) than among responders ( 6 of $11,55 \%)$. Patients taking concomitant SCBs had a higher ratio of adverse effects ( 5 of $16,31 \%)$ than their counterparts (1 of 10, 10\%).

Conclusion: Lacosamide is an effective and tolerable antiepileptic drug in adolescents with LGS. Concomitant SCB use may lead to less effective treatment and more adverse events.

Keywords: Lacosamide; Lennox Gastaut syndrome; Epilepsy; Sodium channel blockers

\section{Introduction}

Lennox-Gastaut syndrome (LGS) is a type of epileptic encephalopathy with childhood onset. It is characterized by drug-resistant seizures with multiple seizure types, delayed development, and characteristic electroencephalography (EEG) patterns of generalized slow spike-wave discharges or general-

Copyright @ 2020 Korean Child Neurology Society

This is an Open Access article distributed under the terms of the Creative Commons Attribution Non-Commercial License (http://creativecommons.org/licenses/by-nc/4.0/) which permits unrestricted non-commercial use, distribution, and reproduction in any medium, provided the original work is properly cited. 
ized paroxysmal fast activities. About $1 \%$ to $2 \%$ of epilepsy patients are diagnosed with LGS [1]. Seizures are often resistant to diverse medications and adjuvant therapies such as ketogenic diet, corpus callosotomy, resective surgery, or vagus nerve stimulation (VNS) [2].

Lacosamide (LCM) is an antiepileptic drug (AED) which augments slow inactivation of voltage-gated sodium channels and stabilizes neuronal membrane [3]. The medication was initially approved for treatment of focal (partial-onset) epilepsy for patients over 16 years (Europe, Korea) or 17 years old (United States). The novel mechanism of LCM led to research on efficacy of LCM for seizure control in patients with focal epilepsy and drug-resistant epilepsy, including pediatric patients. Several research centers investigated possible interaction between LCM and other AEDs with sodium channel blocking properties, showing mixed results.

Since the United States Food and Drug Administration (USFDA) approved of pediatric LCM use recently in 2017, there are limited data on treatment of LCM on LGS patients. We aimed to assess the efficacy and safety of LCM on Korean adolescent LGS population in a single tertiary center. Because most LGS patients are on multiple AEDs including sodium channel blockers (SCBs), we also sought to assess the effect of LCM and concomitant use of other SCBs.

\section{Materials and Methods}

We performed a retrospective study of 26 patients who were diagnosed with LGS and started LCM as adjuvant antiepileptic medication at a single tertiary referral center between March 2014 and June 2019. We initially reviewed medical records of 53 adolescents who were diagnosed with LGS a tried LCM. Among them, we filtered 36 patients who started LCM from age 16 to 18 . We excluded four patients who maintained LCM for less than 3 months, one patient who had tuberectomy at 2 months after LCM add-on, which may blur the effect of LCM, and five patients who had incomplete information on seizure frequency before or after LCM use, leaving 26 patients who maintained LCM and visited the clinic for at least 3 months after LCM add-on. Review of medical records was done from September 2019 to December 2019.

Patients were diagnosed with LGS by clinical symptoms and EEG. They had intellectual disability and drug-resistant epilepsy with multiple semiology. EEG at initial diagnosis showed patterns of slow and disorganized background with multifocal sharp wave discharges, generalized slow spike wave discharges and/or generalized paroxysmal fast activities [1].

For etiologic evaluation, all patients had gone through brain magnetic resonance imaging (MRI). If there was no clinical history of possible acquired etiology, targeted gene panel for epilepsy com- prising 172 genes related to epilepsy had been tested.

Other clinical history included age, sex, seizure semiology, frequency and intensity, and previous treatment before LCM initiation. Adjuvant treatment comprised of ketogenic diet, VNS, or epileptic surgeries including corpus callosotomy, disconnection, or resection of possible lesion. We noted concomitant AEDs including other SCBs.

Patients had started LCM from daily dosage of 1.5 to $4 \mathrm{mg} / \mathrm{kg}$ divided in two doses. Dosage had been titrated up to $12.5 \mathrm{mg} / \mathrm{kg} /$ day at most, not exceeding $600 \mathrm{mg} /$ day. All patients visited the clinic over 6 months after LCM add-on. Every 3 to 6 months, we checked seizure frequency, intensity and drug-related adverse events. We defined 'responders' as patients whose seizure frequency decreased more than 50\% of baseline after taking LCM for 3 months. If adverse events related to LCM were not negligible, slow titration, transient tapering, or transient discontinuation was done. After symptoms subsided, dosage was gradually resumed.

Statistic evaluation was done with chi-square tests, exact Fisher's tests, and Mann-Whitney U tests using SPSS version 23.0 (IBM Co., Armonk, NY, USA). This study was approved by Institutional Review Board (IRB) in Severance hospital, Seoul, Korea (IRB protocol number 4-2020-0292). Written informed consent by the patients was waived due to a retrospective nature of our study.

\section{Results}

Our study included 26 adolescents diagnosed with LGS (Table 1). Among them, 17 patients were male (65\%). Patients had seizure onset at median 2.0 years of age (interquartile range [IQR], 0.7 to 4.3). Most common etiology was congenital structural, found in seven patients (27\%) with focal cortical dysplasia, heterotopia, and pachygyria (Table 1). Six patients (23\%) had acquired structural etiology of periventricular leukomalacia, traumatic brain injury, injury after chemotherapy and radiotherapy, and hypoxic injury after a major operation. Six patients had infectious etiology (23\%) which presented as encephalitis, bacterial meningitis and neonatal sepsis. One patient (4\%) had genetic etiology of ZEB2 gene mutation. The remaining six patients (23\%) had no etiology found after extensive work-up.

Patients added LCM at median 17.1 years of age (Tables 1 and 2). At LCM initiation, patients were on median four antiepileptic medications (IQR, 3 to 4). A majority of 23 patients (88\%) tried adjunctive therapies. Among them, 18 patients (69\%) had tried ketogenic diet or modified Atkins' diet. While 16 patients had stopped dietary therapies mostly due to lack of seizure control, poor compliance, or onset of systemic or gastrointestinal disease, two patients were still on modified Atkins' diet at the time of LCM 
Table 1. Baseline demographics in adolescents with Lennox-Gastaut syndrome $(n=26)$, and comparison between lacosamide responders and non-responders: onset, etiology, treatment history, and lacosamide treatment

\begin{tabular}{lcccc}
\hline Characteristic & Seizure control 50\%-99\% $(n=11)$ & Seizure control <50\% $(n=15)$ & Total $(n=26)$ & P value \\
\hline Male/female (ratio) & $7 / 4(64: 36)$ & $10 / 5(67: 33)$ & $17 / 9(65: 35)$ & 1.000 \\
Age of seizure onset (yr) & $2.0(0.5-3.0)$ & $3.0(0.7-7.0)$ & $2.0(0.7-4.3)$ & 0.526 \\
Etiology (n/subgroup) & & & & \\
$\quad$ Structural (congenital) & $2 / 11(18)$ & $5 / 15(33)$ & $7(27)$ & 0.658 \\
Structural (acquired) & $2 / 11(18)$ & $4 / 15(27)$ & $6(23)$ & 1.000 \\
Genetic & $1 / 11(9)$ & $0 / 15(0)$ & $1(4)$ & 0.423 \\
Infection & $4 / 11(36)$ & $2 / 15(13)$ & $6(23)$ & 0.348 \\
$\quad$ Unknown & $2 / 11(18)$ & $4 / 15(27)$ & $6(23)$ & 1.000 \\
Seizure type & & & & \\
$\quad$ Focal only & $2 / 11(18)$ & $7 / 15(47)$ & $9(35)$ & 0.217 \\
Generalized & $3 / 11(27)$ & $5 / 15(33)$ & $8(31)$ & 0.658 \\
$\quad$ Focal and generalized & $6 / 11(55)$ & $3 / 15(20)$ & $9(35)$ & 0.103 \\
Concomitant AEDs & $4(3-5)$ & $4(3-4)$ & $4(3-4)$ & 0.797 \\
Previous dietary, neuromodulatory, & $10 / 11(91)$ & $13 / 15(87)$ & $23(88)$ & 1.000 \\
or surgical treatment (n/subgroup) & & & & \\
Prior KD & $7 / 11(63)$ & $11 / 15(73)$ & $18(69)$ & 0.683 \\
Prior VNS & $3 / 11(27)$ & $7 / 15(47)$ & $10(38)$ & 0.428 \\
Prior epileptic surgery & $5 / 11(45)$ & $7 / 15(47)$ & $12(46)$ & 0.951 \\
Age at LCM initiation (yr) & $17.1(16.7-17.6)$ & $17.3(16.6-17.9)$ & $17.1(16.7-17.9)$ & 0.511 \\
Maximum LCM dose (mg/kg/day) & $8.0(6.9-10.0)$ & $7.8(6.5-9.8)$ & $8.1(6.8-9.8)$ & 0.701 \\
Duration of LCM (mo) & $21.0(12.0-30.0)$ & $11.0(7.0-15.0)$ & $13.5(7.8-22.5)$ & 0.026 \\
LCM discontinuation & 0 & $7(47)$ & $7(27)$ & 0.010 \\
Concomitant SCB(s) & $6(55)$ & $10(67)$ & $16(62)$ & 0.689
\end{tabular}

Values are presented as median (interquartile range) or number (\%).

AED, antiepileptic drug; KD, ketogenic diet; VNS, vagus nerve stimulation; LCM, lacosamide; SCB, sodium channel blocker.

add-on. Also, 10 patients (38\%) had VNS, and 12 patients (46\%) went through epileptic surgeries including corpus callosotomy, disconnection or resection.

Patients were on LCM for median 13.5 months (IQR, 7.8 to 22.5). After dosage titration, patients' maximum dose was median $8.1 \mathrm{mg} / \mathrm{kg} /$ day (IQR, 6.8 to 9.8 ). Among 26 patients, 22 patients (85\%) continued LCM over 6 months. Eleven patients (42\%) had seizures decreased over 50\% after LCM (Fig. 1). There was no case of seizure freedom.

Among 15 non-responders, eight patients maintained LCM until last visit, for median 15 months (IQR, 11.5 to 21.5). Seven patients withdrew LCM after median 7 months (IQR, 3 to 9) of LCM administration because of poor seizure control, including one patient who had more frequent seizures after LCM add-on. The patient with worsening seizures was a 17-year-old boy who was born at term without perinatal history. He had delayed speech onset at 3 years old, could not walk independently, and started head drop seizures at 11 years old. Brain MRI showed mild enlargement of the ventricles with white matter thinning, suggestive of periventricular leukomalacia. EEG at 17 years of age showed generalized slow spike-waves, and he started LCM while taking topiramate and levetiracetam. He had average 10 head drop seizures noted every week at time of LCM add-on, but after 3 months, he had over 20 weekly seizures.

There was no statistical difference in the previous treatment history and LCM treatment profile between the responders and non-responders (Table 1). Both groups of 11 responders and 15 non-responders to LCM had early onset of seizures, the responders at median age 2.0 years old (IQR, 0.5 to 3.0) and non-responders at age 3.0 years old (IQR, 0.7 to 7.0 ). Both groups started LCM at median age 17.1 years old and were on median four antiepileptic medications including valproate, clobazam, perampanel, and/or SCBs such as lamotrigine, carbamazepine or oxcarbazepine at LCM initiation. Most commonly used AEDs were valproate and lamotrigine (each 14 patients, 54\%), followed by clobazam (10 patients, 38\%).

Higher ratio of non-responders had tried adjunctive therapies compared to responders. Eleven out of 15 non-responders (73\%) had tried ketogenic diet compared to seven out of 11 responders (64\%). Seven of 15 non-responders (47\%) had VNS insertion 
Table 2. Effects of other sodium-channel blockers in the treatment of seizures with lacosamide and lacosamide-related adverse events

\begin{tabular}{|c|c|c|c|}
\hline Variable & SCB (+) & SCB $(-)$ & $P$ value \\
\hline Patients & $16(62)$ & $10(38)$ & - \\
\hline Responders/subgroup & $6 / 16(38)$ & $5 / 10(50)$ & 0.689 \\
\hline LCM-related AEs (n/subgroup) & $5 / 16(31)$ & $1 / 10(10)$ & 0.352 \\
\hline LCM transiently decreased or discontinued after AE & 1 & 1 & 1.000 \\
\hline LCM decreased or discontinued for the rest of the study after AE & 2 & 0 & 0.508 \\
\hline \multicolumn{4}{|l|}{ Clinical manifestation of LCM-associated AEs } \\
\hline Hyperactivity, inattention & 1 & $1^{\mathrm{a}}$ & 1.000 \\
\hline Somnolence & $1^{b}$ & 0 & 1.000 \\
\hline Ataxia & $1^{b}$ & 0 & 1.000 \\
\hline Emesis & $2\left(1^{\mathrm{a}}\right)$ & 0 & 0.508 \\
\hline
\end{tabular}

Values are presented as number (\%).

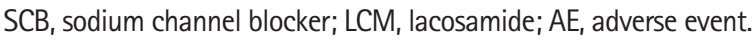

${ }^{a}$ Transient LCM reduction or discontinuation after onset of the adverse event; ${ }^{b}$ LCM reduction or discontinuation for the rest of the study period after onset of the adverse event.

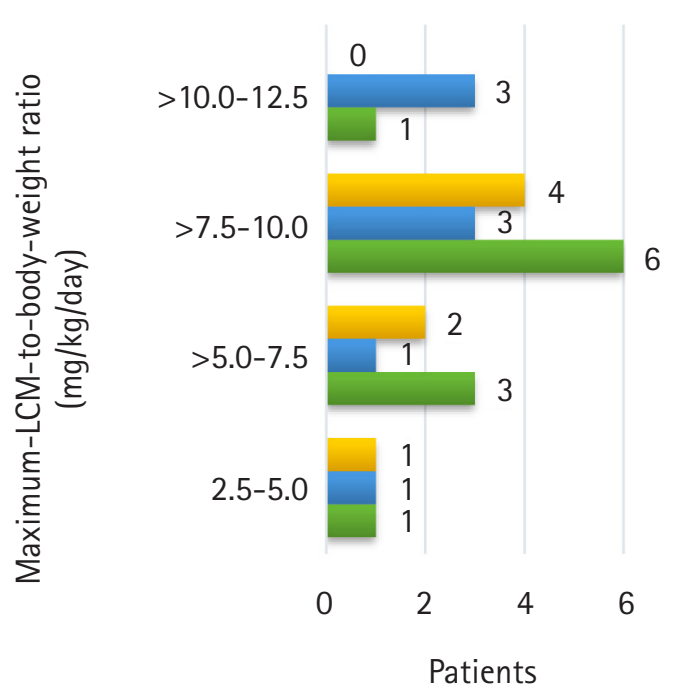

Withdrawal

Seizure control<50\%

Seizure control 50\%-99\%

Fig. 1. Patients' response to lacosamide (LCM) based on LCM dosage per body weight. After dosage titration, patients' maximum dose ranged from 2.6 to $12.5 \mathrm{mg} / \mathrm{kg} /$ day (median, 8.1 [interquartile range, 6.8 to $9.8 \mathrm{mg} / \mathrm{kg} /$ day]). There was no significant difference in dosage per body weight.

compared to three of 11 responders (27\%). Half of the patients in both groups had gone through epileptic surgeries before LCM trial (five of 11 responders [45\%] and seven of 15 non-responders [47\%], respectively). Responders had median maximum-LCM-tobody-weight ratio of $8.0 \mathrm{mg} / \mathrm{kg} /$ day (IQR, 6.9 to 10.0) and non-re-

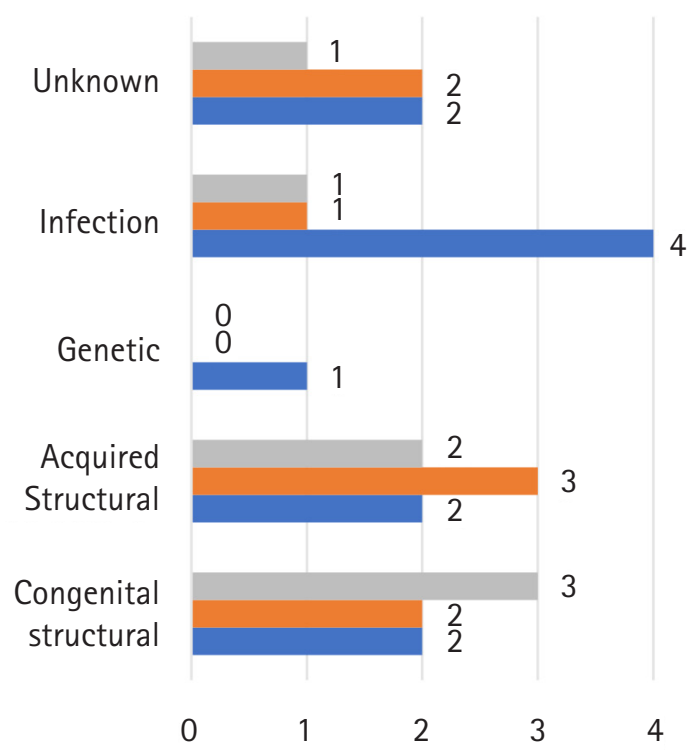

Withdrawal

Seizure control<50\%

- Seizure control 50\%-99\%

Fig. 2. Patients' response to lacosamide based on etiology. There was no statistically significant difference in the response or withdrawal rate according to etiology.

sponders had median ratio of $7.8 \mathrm{~kg} / \mathrm{mg} /$ day (IQR, 6.5 to 9.8 ).

There was no statistically significant difference in efficacy of LCM among all etiologic categories (Table 1, Fig. 2). The patient with ZEB2 gene mutation responded to LCM. Among six patients with infectious causes, four patients responded to LCM. One-third of the patients responded to LCM in the groups with congenital structural, acquired structural, and unknown origin (two patients each). On the other hand, three out of seven patients with congeni- 
tal structural lesions withdrew from LCM due to poor seizure control. Two out of six patients with acquired structural lesions, one patient with infectious etiology and one patient with unknown etiology also stopped LCM because of little effect.

Among 11 responders, six patients (55\%) were on other SCBs such as lamotrigine, carbamazepine, or oxcarbazepine at the time of LCM add-on. On the other hand, 10 out of 15 non-responders (66\%) were on SCBs. There was no patient with phenytoin during LCM trial.

LCM-related adverse events were noted in six patients (23\%) (Table 2). There were two cases of behavioral changes such as hyperactivity and inattention, and two cases of emesis. Other two cases were somnolence and ataxia. Two patients required transient decrease or discontinuation of LCM, but resumed the dosage after symptoms subsided. Two non-responders, one with somnolence and another with ataxia, reduced LCM for the rest of the study period. No patient discontinued LCM solely because of adverse events.

There were five cases of adverse events in 16 patients with concomitant SCBs (27\%) (Table 2). In the two cases of somnolence and ataxia, LCM was decreased for the rest of the study period. Also, in another case of emesis, LCM was transiently discontinued. In 10 patients without concomitant SCBs, there was only one case of adverse event (18\%). The patient had emesis, and had transient reduction of LCM. The results were statistically insignificant.

\section{Discussion}

Since the US-FDA and European Commission (EC) approved of LCM in 2008 for treatment of epilepsy in patients 16 years (EC) or 17 years (US-FDA) older [3], previous studies on LCM reported its efficacy and safety on focal epilepsy or drug-resistant epilepsy mainly in adults. However, due to the medication's unprecedented mechanism of sodium-channel blocking effect by enhancing slow activation of voltage-gated sodium channels, pediatric neurologists cautiously scrutinized its effects on drug-resistant epilepsy [4-6]. Guilhoto et al. [4] reviewed LCM treatment in 16 pediatric patients and found six responders (37.5\%) with four cases $(25 \%)$ of non-severe adverse events which prompted the discontinuation of LCM. Grosso et al. [6] performed a prospective multi-center study of LCM use in 24 pediatric patients with focal epilepsy under age 4 and found 10 responders after 3 months of LCM (42\%), including four patients who became seizure free (17\%). After 12 months, four patients (22\%) were still responding to LCM. Adverse effects were non-severe and found in eight patients (33\%) [6]. In light of accumulating reports on pediatric population, in 2017, the USFDA allowed of extrapolating oral LCM use for treatment of focal epilepsy on pediatric population aged 4 and older [7].

Since LGS has onset age at childhood, there were scarce studies on LGS before the recent FDA allowance of LCM. Rastogi and Ng [5] prospectively studied efficacy of LCM on 16 pediatric patients, including those with diagnosis of LGS. Among the four LGS patients, two patients had seizures decreased over $90 \%$, and two patients had no effect [5]. Andrade-Machado et al. [8] reported a case of 20-year-old LGS patient who had seizures and EEG patterns worsened after LCM usage and returned to baseline after its discontinuation. Grosso et al. [9] suggested possible efficacy of LCM on a multi-center retrospective study of 18 children with LGS, of whom six patients (33\%) responded to LCM. Miskin et al. [10] reported efficacy of LCM on 21 pediatric patients with drug-resistant generalized epilepsy, eight of whom had LGS, and seven of the eight patients (87.5\%) responded to LCM.

Our study includes 26 patients with LGS, the largest cohort of adolescents with LGS who tried LCM. In our cohort, 11 of 26 patients (42\%) responded to LCM, which is comparable to above previous reports of variable drug response in LGS which ranged from $33 \%$ to $88 \%[5,9,10]$. There was no case of seizure freedom, which is consistent with the three studies. Adverse effects were noted on six patients (25\%), which were not severe. This result is less than previous report of non-severe adverse events of $44 \%$ in LGS [9], and results of other pediatric studies, which ranged from $29 \%[10]$ to $51 \%[11]$.

Whether LCM and SCBs work synergistically or additively remains unknown. The fact that LCM enhances slow inactivation of voltage-gated sodium channels, while other SCBs act on fast inactivation [12] suggest that these drugs may work synergistically. However, previous studies have been controversial, and some were against the concomitant use of SCBs and LCM. A study on 158 epilepsy patients with mean age of 42.1 years demonstrated higher efficacy and lower adverse events in patients without concomitant SCBs compared to patients with concomitant SCBs [13]. A more recent study evaluated LCM retention rates in 223 pediatric drug-resistant epilepsy patients, and found that LCM treatment failure was correlated with concomitant treatment of SCBs [14]. On the other hand, one study noted that among 21 pediatric patients with focal epilepsy treated with LCM, there was no significant difference in efficacy or adverse effects between those with simultaneous use of SCBs and others without SCBs [15].

Although statistically insignificant, the results of our study suggest possible interaction of LCM with concomitant SCBs. More patients without concomitant SCBs responded to LCM (six out of $11,55 \%$ ) compared to patients with concomitant SCBs (five out of $15,33 \%)$. Patients without other SCBs also had lower percentage of adverse effects ( 1 of $10,10 \%)$ compared to those with other 
SCBs (five of 16, 31\%). Also, more patients with concomitant SCBs had adverse events requiring LCM decrease during the rest of study period (two cases), or transient discontinuation of LCM (one case), compared to only one case in non-SCB group presenting emesis who had transient reduction of LCM.

The mechanisms of the possible interaction between LCM and other SCBs are under investigation. The report that plasma concentrations of lamotrigine and oxcarbazepine were not affected by concomitant LCM use suggest that effects of LCM on these drug concentrations is minimal [16]. Another report indicates a low potential for pharmacokinetic drug-drug interaction between LCM and carbamazepine [17]. Novy et al. [18] suggested there may be pharmacodynamic interaction between LCM and SCBs, as the AED levels do not change during the intolerable adverse events. Further studies would give more precise guideline on prescription of LCM and other SCBs.

Our study adds to rare studies specifying LCM use in pediatric LGS population. Because patients with drug-resistant epilepsy are on hardship of taking multiple AEDs usually twice a day, our information on concomitant SCB use may prevent some patients from excessive periods of inefficacy and intolerability by LCM and concomitant SCBs. However, our data is based on retrospective chart review, so some of the medical records may not contain information on efficacy or adverse effects of LCM. We could include a small number of 26 patients due to low prevalence of LGS in adolescents and short period of LCM use in pediatric patients, and a larger cohort may enable a statistically significant evaluation. We evaluated patient response at 3 months of LCM use, but since patients with drug-resistant epilepsy take AEDs for years, more information on long-term use could give pragmatic clinical information. Although our patients took oral tablets of LCM which were the only option in Korean market during the study period, further studies on intravenous LCM use in pediatric patients may help them in cases when they cannot tolerate oral medications or when they need fast activation of the medication.

In conclusion, LCM is an effective and tolerable medication for seizure control in adolescents with LGS. Concomitant use of SCBs could lead to decreased effect or increased adverse events of LCM. Further studies on LCM in pediatric population could enhance proper treatment with LCM in LGS patients.

\section{Conflicts of interest}

No potential conflict of interest relevant to this article was reported.

\section{ORCID}

Han Som Choi, https://orcid.org/0000-0002-5818-7985

Se Hee Kim, https:/ orcid.org/0000-0001-7773-1942

Heung Dong Kim, https:/ / orcid.org/0000-0002-8031-7336

\section{Author contribution}

Conceptualization: SHK and HDK. Data curation: HSC, HCK, and HDK. Formal analysis: HSC and SHK. Methodology: HSC and SHK. Project administration: HSC, SHK, and JSL. Visualization: HSC and SHK. Writing - original draft: HSC. Writing - review \& editing: HSC, SHK, HCK, JSL, and HDK.

\section{References}

1. Asadi-Pooya AA. Lennox-Gastaut syndrome: a comprehensive review. Neurol Sci 2018;39:403-14.

2. Mastrangelo M. Lennox-gastaut syndrome: a state of the art review. Neuropediatrics 2017;48:143-51.

3. Perucca E, Yasothan U, Clincke G, Kirkpatrick P. Lacosamide. Nat Rev Drug Discov 2008; 7:973-4.

4. Guilhoto LM, Loddenkemper T, Gooty VD, Rotenberg A, Takeoka M, Duffy FH, et al. Experience with lacosamide in a series of children with drug-resistant focal epilepsy. Pediatr Neurol 2011;44:414-9.

5. Rastogi RG, Ng YT. Lacosamide in refractory mixed pediatric epilepsy: a prospective add-on study.J Child Neurol 2012;27:492-5.

6. Grosso S, Parisi P, Spalice A, Verrotti A, Balestri P. Efficacy and safety of lacosamide in infants and young children with refractory focal epilepsy. Eur J Paediatr Neurol 2014;18:55-9.

7. Practice guideline update summary: efficacy and tolerability of the new antiepileptic drugs II: treatment-resistant epilepsy. Report of the Guideline Development, Dissemination, and Implementation Subcommittee of the American Academy of Neurology and the American Epilepsy Society. Neurology 2018;91:1117.

8. Andrade-Machado R, Benjumea-Cuartas V, Jaramillo-Jimenez E. Lacosamide in Lennox-Gastaut syndrome: case report. Clin Neuropharmacol 2012;35:148-9.

9. Grosso S, Coppola G, Cusmai R, Parisi P, Spalice A, Foligno S, et al. Efficacy and tolerability of add-on lacosamide in children with Lennox-Gastaut syndrome. Acta Neurol Scand 2014;129:420-4.

10. Miskin C, Khurana DS, Valencia I, Legido A, Hasbani DM, Carvalho KS. Efficacy and tolerability of lacosamide in the treatment of children with refractory generalized epilepsy. J Child Neurol 2016;31:925-8.

11. Pasha I, Kamate M, Didagi SK. Efficacy and tolerability of lacos- 
amide as an adjunctive therapy in children with refractory partial epilepsy. Pediatr Neurol 2014;51:509-14.

12. Errington AC, Stohr T, Heers C, Lees G. The investigational anticonvulsant lacosamide selectively enhances slow inactivation of voltage-gated sodium channels. Mol Pharmacol 2008;73:157-69.

13. Villanueva V, Lopez-Gomariz E, Lopez-Trigo J, Palau J, Garcia $\mathrm{M}$, Villarroya T, et al. Rational polytherapy with lacosamide in clinical practice: results of a Spanish cohort analysis RELACOVA. Epilepsy Behav 2012;23:298-304.

14. McGinnis E, Kessler SK. Lacosamide use in children with epilepsy: retention rate and effect of concomitant sodium channel blockers in a large cohort. Epilepsia 2016;57:1416-25.

15. Kim JS, Kim H, Lim BC, Chae JH, Choi J, Kim KJ, et al. Lacosamide as an adjunctive therapy in pediatric patients with refrac- tory focal epilepsy. Brain Dev 2014;36:510-5.

16. Steinborn B, Stockis A, Zhang Y, Dimova S, Martin P. Lack of pharmacokinetic interaction of lacosamide on carbamazepine, lamotrigine, levetiracetam, oxcarbazepine, topiramate and valproic acid in children and adolescents with epilepsy: post-hoc analysis of a randomized, double-blind, placebo-controlled trial (P4. 270). Neurology 2018;90(15 Suppl):P4.270.

17. Cawello W, Nickel B, Eggert-Formella A. No pharmacokinetic interaction between lacosamide and carbamazepine in healthy volunteers. J Clin Pharmacol 2010;50:459-71.

18. Novy J, Patsalos PN, Sander JW, Sisodiya SM. Lacosamide neurotoxicity associated with concomitant use of sodium channel-blocking antiepileptic drugs: a pharmacodynamic interaction? Epilepsy Behav 2011;20:20-3. 